\title{
Research of Tire Rolling Radius Based On the Finite Vibration Unit Model
}

\author{
Wang Liqiang, Han Zongqi, Liu Quanyou, Xu Yunxiao, Bao Qianqian \\ College of Vehicle and energy, Yanshan University, Qinhaungdao, Hebei, China, 066004
}

\begin{abstract}
This paper established the finite vibration unit model and solved the response of each unit system. And it analyzed the unit vibration. Based on the model it introduced the calculation method of rolling radius. Next this paper simulated rolling radius and the relationships between the rolling radius and the vehicle speed, the tire pressure, and the load using MATLAB software. And the relationships were modified according to the real vehicle test. Finally, the tire rolling radius calculation method was discovered at high speed.
\end{abstract}

Key Words: The finite vibration unit model; rolling radius; simulation; the real vehicle test

\section{INTRODUCTION}

Tire is an essential part of the vehicle. It supports the whole vehicle and develops longitudinal forces for acceleration and braking and lateral forces for cornering, while cushioning against road shocks and vibration under the interaction with suspension. Tire model is one of the most important models of vehicle dynamics so that the scholars all over the world have conducted a careful study on it ${ }^{[1]}$. Generally, in the process of modeling, the tire is simplified into a series of ideal unit with the given physical properties. If necessary, the sliding ability of these units on the road surface also can be given as well as the mutual constraint between adjacent units ${ }^{[2]}$. More attractive theoretical model due to its generality characteristics has been the mainstream of the research in this field and the striving direction.

Incentives for the tire from road have two aspects. One is radial incentives which form because of tire continuous local compression and release through the contact face; the other is longitudinal incentives which form because of continuous rolling and release between road and tire rubber ${ }^{[3]}$. Generally, incentives from rough surfaces are greater than that from flat surfaces. These incentives acting on tire form longitudinal force、 lateral force and radial force, sometimes even a moment. This paper mainly studies the effect of radial incentive on the tire rolling radius.

\section{THE FINITE VIBRATION UNIT MODEL}

Tire is an elastomer of quality which can be simulated using a series of radial spring and damper. The contact area between the tire and road in the longitudinal direction is far greater than the circumferential size of the tire unit in the circumferential direction ${ }^{[4]}$. Therefore, the effect of the adjacent unit on radial vibration can be ignored. The tire model is simplified as shown in Fig.1.

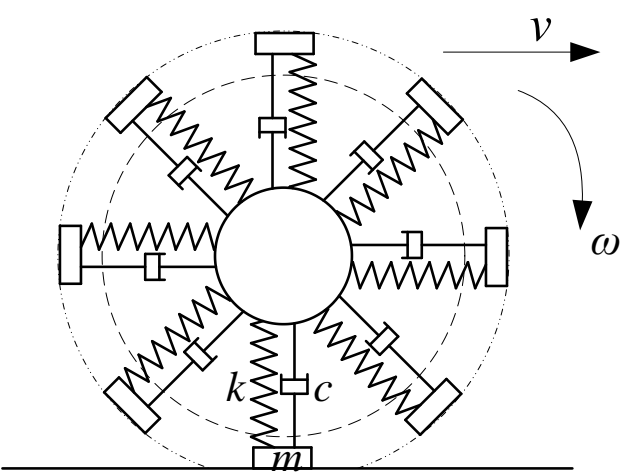

Fig. 1 The finite vibration unit tire model

The tire is simplified into $\mathrm{n}$ units that are uniformly distributed along the circumferential direction, and the quality of these $\mathrm{n}$ units are $\mathrm{m}(\mathrm{m}=\mathrm{M} / \mathrm{N}$, where $\mathrm{M}$ is the quality of tire). Each unit is connected to the rim with a spring whose elastic coefficient is $\mathrm{K}$. The damping coefficient of unit along the radial vibration is $\mathrm{C}$. These units are independent of each other.

On a hard straight road, tire is in the steady state of pure rolling, no offset and slip. Supposing the speed is $v$, and the free radius of tire is $r_{0}$. The changes of the rolling radius of tire are ignored. So the time needed for 
tire rolling a circle is $T=2 \pi r_{0} / v$. In the rolling process, each unit contact with the road in turn. Thus each unit suffers shock at regular intervals $T$ from road. The duration of each shock is $t_{0}=T / n$ and the size of the force is $P=F_{z}$, where $F_{z}$ is the load of a tire. The impulse function is $P(t)$, as:

$$
\mathrm{P}(\mathrm{t})= \begin{cases}P & \left(0 \leq t \leq t_{0}\right) \\ 0 & \left(t_{0} \leq t \leq T\right)\end{cases}
$$

Any unit can be regarded as a single degree-of-freedom damping vibration system as shown in Fig.2. The system is subjected to a step cycle impact force ignoring the time needed for load increasing from zero to the value of $P$ as shown in Fig.3.

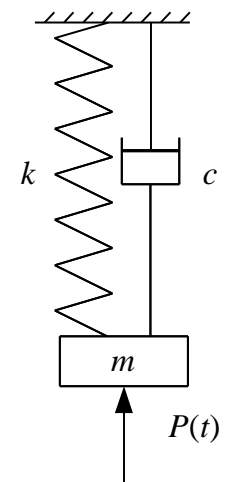

Fig.2 Unit vibration system

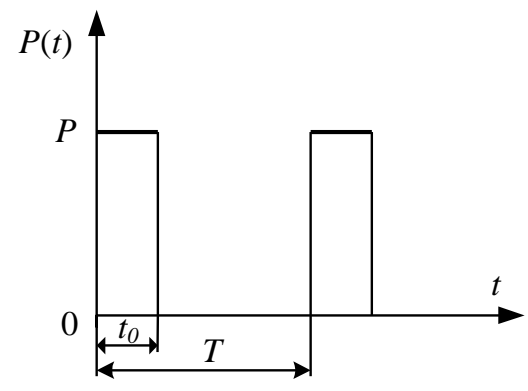

Fig.3 Impact force $P(t)$

\section{RESPONSE OF A SINGLE DEGREE-OF-FREEDOM SYSTEM}

Impulse response function is used to analyze the response of a system under the action of the unsteady incentive. The impulse response function is defined as transient response of the system under the action of unit impulse force. The impulse response function can be further defined as transient response $h(t)$ generated by pulse per unit force (or unit impulse force) at $\mathrm{t}=0$ for the system in the stationary state originally ${ }^{[5-6]}$. Obviously, at $t<0, h(t)$ equals zero.

The differential equation of motion for a single degree-of-freedom system is supposed as:

$$
m \ddot{x}+c x+k \dot{x}=\delta(t)
$$

The free attenuation vibration response for a single degree-of-freedom linear system with weakly damped is shown as follow:

$$
x=A e^{-\varepsilon p t} \sin (i q t+\varphi)
$$

Where:

$p=\sqrt{k / m}$ Natural frequency of the vibration system

$\varepsilon=\mathrm{c} / 2 p m$ Damping ratio of the vibration system

$q=\sqrt{1-\varepsilon^{2}} p$ Damping natural frequency of the vibration system

If a unit impulse force is acted at $\mathrm{t}=\mathrm{T}$, the response of the system should be as follow:

$$
x(t)=h(t-\tau)=\left\{\begin{array}{cc}
\frac{1}{m q} e^{-\varepsilon p(t-\tau)} \cdot \sin q(t-\tau), & t>\tau \\
0, & t<\tau
\end{array}\right.
$$

The total response of the system corresponding to the $f(t)$ is as follow:

$x(t)=\int_{0}^{t} h(t-\tau) f(t) d \tau$

So the response of the system to arbitrarily excitation with zero initial conditions can be expressed with convolution integral of pulse response and the excitation. The response with zero initial conditions after plugging $h(t-\tau)$ in into equation (5) is as follow:

$$
x(t)=\frac{1}{m q} \int_{o}^{t} f(\tau) e^{-\varepsilon p(t-\tau)} \cdot \sin q(t-\tau) d \tau
$$

If at the beginning of the exciting force at $\tau=0$, the quality has initial displacement and initial velocity, the general solution for the system is as follow:

$$
x(t)=e^{-\varepsilon p t}\left(x_{0} \cos q t+\frac{\varepsilon p x_{0}+x_{0}}{q} \sin q t\right)+\frac{1}{m q} \int_{o}^{t} f(\tau) e^{-\varepsilon p(t-\tau)} \sin q(t-\tau) d \tau
$$

\section{Vibration analysis of unit}

The initial conditions for the system under the effect of impulse are $\mathrm{x}_{0}=0, \dot{x_{0}}=S / m$. The response for the vibration system using the formula (5) is as follow: 


$$
x(t)=e^{-\varepsilon p t}\left(x_{0} \cos q t+\frac{\varepsilon p x_{0}+\dot{x}_{0}}{q} \sin q t\right)+\frac{1}{m q} \int_{o}^{t} P(\tau) e^{-\varepsilon p(t-\tau)} \sin q(t-\tau) d \tau
$$

Where:

$S=P \cdot \Delta t$ Impulse that the units withstand in the contact moment

After plugging the initial conditions of vibration system into the equation (8),the response goes as follow:

$x(t)=\frac{S}{q m} e^{-\varepsilon p t} \sin q t+\frac{P}{k}\left[1-e^{-\varepsilon p t}\left(\cos q t+\frac{\varepsilon p}{q} \sin q t\right)\right]$

The impact that any unit withstands is applied in a very short time in the rolling process. In finite load condition, impulse can be thought of as zero. Thus the equation (9) can be simplified to the equation (10),as:

$x(t)=\frac{P}{k}\left[1-e^{-\varepsilon p t}\left(\cos q t+\frac{\varepsilon p}{q} \sin q t\right)\right]$

After plugging the parameters of vibration system into the equation (10),the response goes as follow:

$x(t)=\frac{F_{Z}}{k}\left[1-2 e^{-\frac{c}{2 m} t}\left(\cos \left(\frac{\sqrt{4 k m-c^{2}}}{2 m} t\right)+\frac{c}{\sqrt{4 k m-c^{2}}} \sin \left(\frac{\sqrt{4 k m-c^{2}}}{2 m} t\right)\right)\right]$

The maximal displacement of the tire units occurs at $\mathrm{t}_{0}=T / n=2 \pi r_{0} / n v$ in the process of contact with the road. At this time, the maximal displacement is $x_{\max }$,as:

$x_{\max }=\frac{F_{Z}}{k}\left[1-2 e^{-\frac{c}{2 m} \cdot \frac{2 \pi r_{0}}{n v}}\left(\cos \left(\frac{\sqrt{4 k m-c^{2}}}{2 m} \cdot \frac{2 \pi r_{0}}{n v}\right)+\frac{c}{\sqrt{4 k m-c^{2}}} \sin \left(\frac{\sqrt{4 k m-c^{2}}}{2 m} \cdot \frac{2 \pi r_{0}}{n v}\right)\right)\right]$

So on the road, the maximum radial deformation for tires is $\Delta r_{\max }$,as:

$\Delta r_{\max }=x_{\max }$

The rolling radius of unit model

When ignoring the tire damping effects on vibration, the maximum radial deformation for tires based on equation (12) and (13) is $\Delta r_{\max }$, as:

$$
\Delta r_{\max }=\frac{F_{z}}{k}\left[1-\cos \left(\sqrt{\frac{k}{m}} \cdot \frac{2 \pi r_{0}}{n v}\right)\right]
$$

Where: $k=\gamma p, k$ is elastic coefficient of finite unit vibration model, $N / \mathrm{mm} . \gamma$ is ratio coefficient which is related to the structure and material of the tire, $\gamma=1.5 \sim 3.5$. At high speed the rolling radius of the tire whose free radius is $r_{0}$ can be expressed as $r_{r}$, as:

$$
r_{r}=r_{0}-\frac{F_{Z}}{\gamma p}\left[1-\cos \left(\sqrt{\frac{\gamma p}{m}} \cdot \frac{2 \pi r_{0}}{n v}\right)\right]
$$

Where:

$r_{r}=$ Tire rolling radius $(m)$

$r_{0}=$ Tire free radius $(m)$

$\mathrm{F}_{\mathrm{z}}=$ Tire load $(N)$

$\mathrm{P}=$ tire pressure $(\mathrm{Pa}), \mathrm{p}=0.5 \times 10^{5} \sim 4.0 \times 10^{5} \mathrm{~Pa}$

$\mathrm{V}=\operatorname{Speed}(\mathrm{km} / \mathrm{h}), \mathrm{v} \geq 35 \mathrm{~km} / \mathrm{h}$

$\mathrm{n}=$ The number of units. In general, the footprint at ground contact area of tire is $4 \sim 17 \mathrm{~cm}$. The value of $\mathrm{n}$ should be chosen from 12 to 45 combining with the tire circumference, while the concrete numerical value depends on the tire structure and material, etc.

$\mathrm{m}=$ The quality of unit $(\mathrm{kg})$. It is determined by the tire quality and the number of units.

Experimental study

A simulation calculation on the rolling radius of the finite vibration unit model is conducted for the tires of HANKOOK 185/70R14 88T. The tire free radius is $307 \mathrm{~mm}$, quality is $7.2 \mathrm{~kg}$, maximum load is $5.5 \mathrm{kN}$, maximum speed is $190 \mathrm{~km} / \mathrm{h}$, ratio coefficient is 2.5 , the number of units is 30 and the quality of each unit is $0.24 \mathrm{~kg}$. When air pressure is $50 \sim 400 \mathrm{kPa}$ and speed is more than $36 \mathrm{~km} / \mathrm{h}$, the tire rolling radius is calculated by the equation (15), as

\section{Correction of speed}

$$
r_{r}=0.307-\frac{0.4 \cdot F_{z}}{p}\left[1-\cos \left(\sqrt{10.4 \cdot p} \cdot \frac{0.064}{v}\right)\right]
$$

The tire rolling radius increases with speed by comparing simulation results with the test results. In the test when the speed is $50 \sim 140 \mathrm{~km} / \mathrm{h}$, the relationship between rolling radius and the speed is linear. This seems inconsistent with the simulation result. But when the speed is $36 \sim 190 \mathrm{~km} / \mathrm{h}$, the relationship between rolling radius and the speed is approximately parabolic. However, when the speed is in the local scope of $50 \sim 140$ $\mathrm{km} / \mathrm{h}$, rolling radius and the speed is close to the linear relationship again. In order to make the calculation model of rolling radius to better reflect the actual situation, the equation (16) is modified to the equation (17), as:

$r_{r}=0.307-0.4 \cdot \frac{F_{z}}{p}\left[1-\cos \left(\sqrt{10.4 \cdot p} \cdot \frac{0.012}{v}\right)\right]+10^{-7} \cdot v^{2}+1.2 \times 10^{-5} \cdot v-0.009$ 
The equation (17) is simulated according to the test conditions of the relationship between rolling radius and speed. The simulation curve is modified as shown in Fig.4. The simulation curve in Figure 4 on the change tendency of the rolling radius with the speed is basically consistent with the test results. It still has a certain deviation in the values, but the maximum deviation is less than $0.5 \mathrm{~mm}$ and the relative error is less than $2 \%$. Therefore, the equation (17) can be used to predict the relationship between rolling radius and speed of HANKOOK $185 / 70 R 1488 \mathrm{~T}$ tire when inflation pressure is $250 \mathrm{kPa}$ and the load is $3500 \mathrm{~N}$.

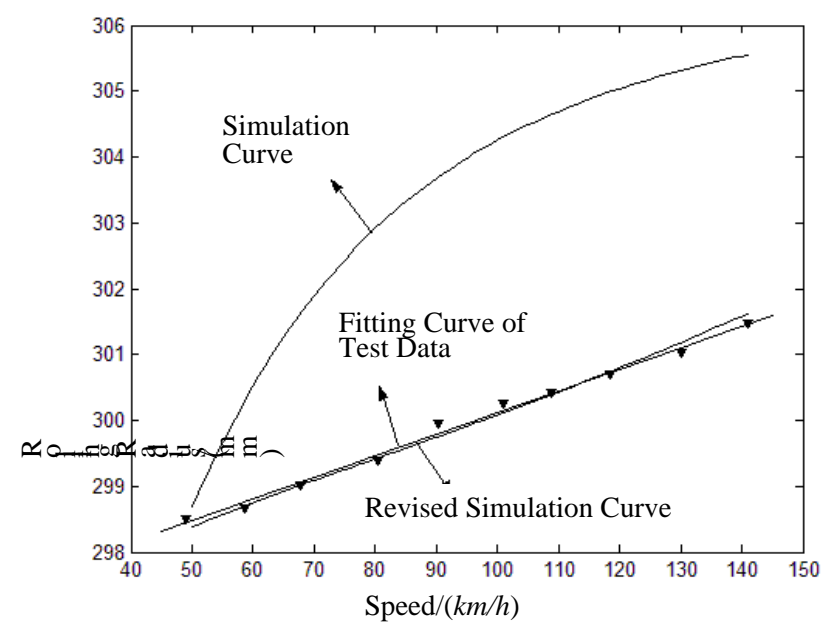

Fig.4 The revised relationship between rolling radius and the speed

\section{Correction of pressure}

Similarly, for more accurate calculation of the tire rolling radius the equation (16) is modified to the equation (18), as:

$$
r_{r}=0.307-0.35 \cdot \frac{F_{Z}}{p}\left[1-\cos \left(\sqrt{12.2 \cdot p} \cdot \frac{0.064}{v}\right)\right]-0.001
$$

The equation (18) is simulated according to the test conditions of the relationship between rolling radius and pressure. The simulation curve is modified as shown in Figure 5. As seen in Fig. 5, the simulation curve in Fig. 5 is basically consistent with the change tendency of test results. And the value is highly consistent. Therefore, the equation (18) can be used to predict the relationship between rolling radius and pressure of HANKOOK $185 / 70 R 1488 \mathrm{~T}$ tire when the load is $3500 \mathrm{~N}$ and the speed is $60 \mathrm{~km} / \mathrm{h}$.

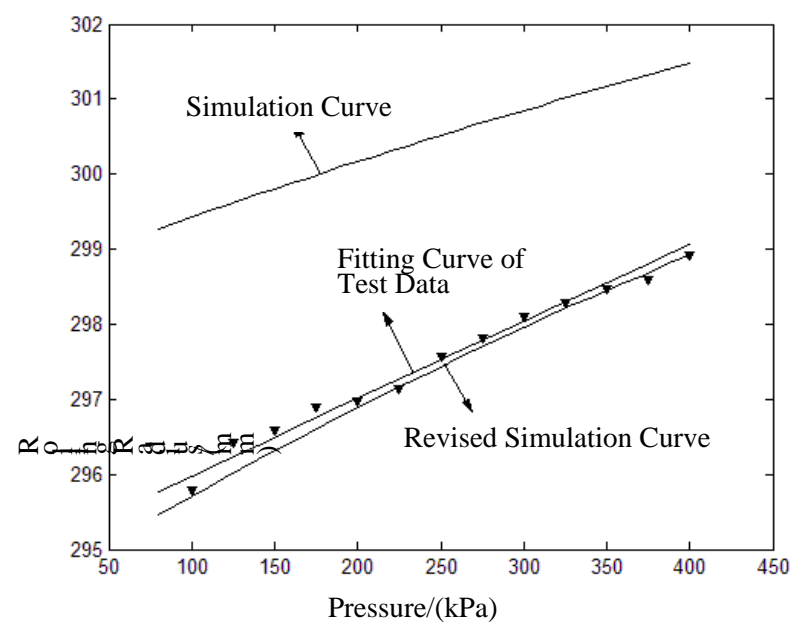

Fig.5 The revised relationship between rolling radius and the pressure

\section{Correction of load}

By the comparison between simulation results and test results, they have many differences in the change of rolling radius with load. So the equation (16) should be modified to the equation (19), as:

$$
r_{r}=0.307-0.04 \cdot \frac{F_{Z}}{p}\left[1-\cos \left(\sqrt{10.4 \cdot p} \cdot \frac{0.064}{v}\right)\right]-0.008
$$


The simulation curve is modified according to the test conditions as shown in Fig.6. The revised simulation curve and test results fit perfectly, and both are highly consistent in value and change trend. Therefore, the equation (19) can be used to predict the relationship between rolling radius and load of HANKOOK 185/70R14 88T tire when inflation pressure is $250 \mathrm{kPa}$, and the speed is $60 \mathrm{~km} / \mathrm{h}$.

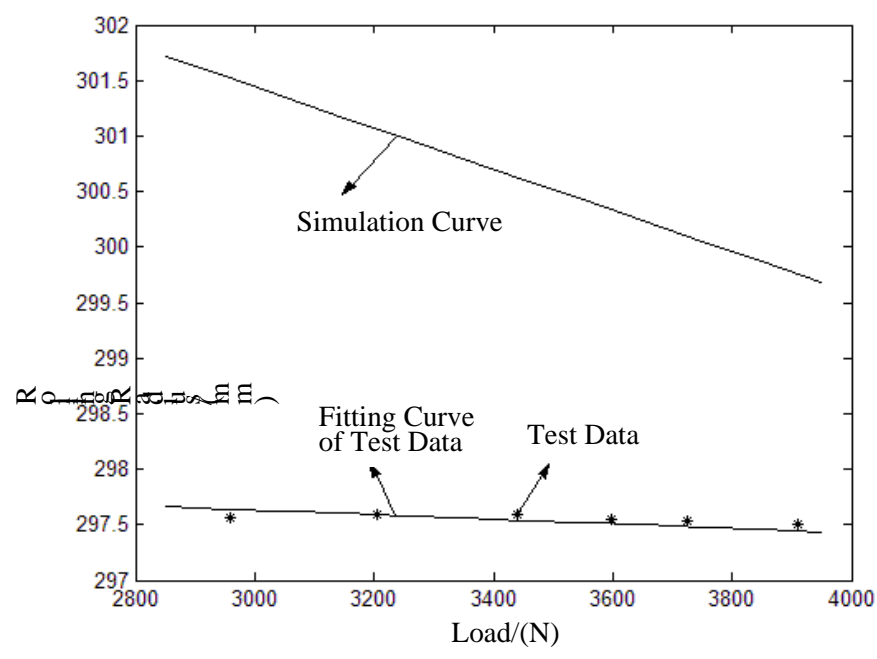

Fig.6 The revised relationship between rolling radius and the load

\section{SUMMARY}

This chapter establishes the finite vibration unit model based on the rolling characteristics of tire. Through the analysis of vibration of the unit, the calculation formula of tire rolling radius and the MATLAB software simulation are conducted. The calculation formula is reasonably modified combining simulation results with real vehicle road test results. The modified formula can accurately calculate the radial tire rolling radius at high speed.

\section{ACKNOWLEDGEMENTS}

This work was financially supported by the National Natural Science Foundation of China (51175450), and Hebei Province Natural Science Foundation (E2012203046).

\section{REFERENCES}

[1] Wang Yupeng, Zhao Longqing. Typical tire models and its development trend. Agricultural equipment and vehicle engineering, 2006,185(12):3-5.

[2] Pang Jian, Zhan Gang, He Hua. Vehicle noise and vibration. Beijing: Beijing Institute of Technology press, 2006:324-328.

[3] Yu Z.X., Tan H.F., Du X.W., and others.A Simple Analysis Method for Contact Defor- mation of Rolling Tire. Vehicle System Dynamics,2001,36(6):435-443.

[4] J.Rcho,K.W.Kim, H. S .Jeong. Numerical Investigation of Tire Standing Wave using 3-D Patterned Tire Model. Journal of Sound and Vibration, 2007,(305):795-807.

[5] Li Huibin. Vibration theory and engineering application. Beijing. Beijing Institute of Technology press, 2006:30-37.

[6] Nedal T. Ratrout. Tire Condition and Drivers' Practice in Maintaining Tires in Saudi Arabia. Accident Analysis and Prevention,2005,37(1):201-206. 